\title{
Nanomechanical Characterization of Single Micron-Sized Polymer Particles
}

\author{
J. Y. He, ${ }^{1}$ Z. L. Zhang, ${ }^{1}$ H. Kristiansen ${ }^{2}$ \\ ${ }^{1}$ NTNU Nanomechanical Lab, Department of Structucal Engineering, Norwegian University of Science and Technology \\ (NTNU), 7491 Trondheim, Norway \\ ${ }^{2}$ Conpart AS, 2013 Skjetten, Norway
}

Received 1 October 2008; accepted 10 December 2008

DOI 10.1002/app.29913

Published online 14 April 2009 in Wiley InterScience (www.interscience.wiley.com).

\begin{abstract}
The mechanical characterization of single micron-sized polymer particles is very important for understanding the anisotropic conductive adhesives interconnection. In this article, a nanoindentation-based flat punch method was employed to investigate the mechanical properties of single polymer particles. A diamond flat tip, instead of a commonly used sharp tip for indentation, was specially designed to deform single polymer particles. The maximum applied load is $10 \mathrm{mN}$ and the linear loading/unloading rate is $2 \mathrm{mN} / \mathrm{s}$. Two types of amorphous
\end{abstract}

polymer particles were examined. The polymer particles display significantly different stress-strain behaviors. The material responses at different strain levels were analyzed and compared. A particle size effect, the smaller the diameter, the harder the particle, on the compression stressstrain behavior, was observed. († 2009 Wiley Periodicals, Inc. J Appl Polym Sci 113: 1398-1405, 2009

Key words: amorphous; crosslinking; mechanical properties; stress; strain

\section{INTRODUCTION}

Polymer particles have received much attention in materials science and pharmaceutical and chemical industries because of their novel characteristics, such as the strong adsorption capability, the surface reactive ability, and the large specific surface area. Examples include carriers for biomolecules in the biomedical field, ${ }^{1}$ the reinforced composite in light concrete, ${ }^{2}$ and the toughening phase in the highimpact polymer materials, ${ }^{3-6}$ etc. Recently, there is a renewed interest in exploiting polymer particles toward use in the manufacturing of electronics and microsystems. One example is the use of metalcoated polymer particles in the anisotropic conductive adhesives (ACA), in which the typical size of particles is from 3 to $10 \mu \mathrm{m}$. The metal-coated polymer particles have potential advantages in terms of reduced package size, of being lead-free, and by reducing manufacturing cost. The substitute of compact metal particles with metal-coated polymer particles improves the compliance of the interconnection and hence enhances the reliability of the assembly. ${ }^{7-12}$ The electrical characteristics as well as

Correspondence to: Z. L. Zhang (zhiliang.zhang@ntnu.no). Contract grant sponsor: Research Council of Norway (through NANOMAT KMB Project); contract grant number: NANOMAT-169737/S10.

Contract grant sponsor: Conpart AS and Invitrogen AS.

Journal of Applied Polymer Science, Vol. 113, 1398-1405 (2009) (c) 2009 Wiley Periodicals, Inc. the reliability of the interconnection are partly determined by mechanical performance of polymer particles. There is also a significant interest for larger metal-coated polymer particles with the diameter of 50 to several hundred microns for use in ball grid arrays and chip scale packaging. In these applications, the added compliance is expected to improve the reliability of the interconnect. There is also a crucial advantage in terms of reduced environmental impact, by reducing the amount of heavy metals. ${ }^{13-15}$ Therefore, the knowledge of mechanical properties of single-polymer and metal-coated polymer particles is of great interest for many potential applications.

Most studies on polymer particles have been focused on synthetic methods and processes. The literature concerning mechanical properties of single particles is relatively sparse. However, mechanical characterization of single particles possesses challenges due to the inherent complexity of the spherical geometry as well as the large deformation involved.

In the past, the mechanical behavior and electrical resistance of ACA assemblies were typically measured through grouping a number of particles (typically several hundreds) between two polished silicon chips. ${ }^{8,16}$ Mostly, ACA assemblies were designed to determine the mechanical and electrical contact properties of the interconnect component including metal-coated polymer particles. The effect of elastic recovery on the electrical contact resistance, the degradation mechanism, and the reliability of ACA 
interconnections were investigated. ${ }^{17,18}$ Nanoindentation with a sharp tip has been used to study mechanical behaviors on the cross-section of polymer particles within the bulk ACA interconnection at various deformation degrees. The aim was to investigate the effect of bonding pressures on the particle properties. It was found that the microhardness at the central area was higher than that at the outside because of the lower constraints at the outside. ${ }^{19,20}$ Subsequently, nanoindentation-based flat punch method was tentatively developed to compress single polymer particles and analyzed ACA performance under different deformation levels. ${ }^{11,21} \mathrm{~A}$ study on mechanical properties of single polymer particles by nanoindentation-based flat punch test was performed. The effects of the swelling ratio and the backbone chain structure on mechanical properties and surface morphologies were investigated. ${ }^{22,23}$ Recently the study on the deformation of single $\mathrm{Ni} / \mathrm{Au}$-coated polymer particles was reported. ${ }^{24}$ The results showed that the particle deformation process was nonlinear and the force/deformation at which particle crushing occurs was affected by the loading rate.

The present work reports the results of an experimental study on single polymer particles under compression. A nanoindentation-based flat punch method was employed to test the particles with two different types of polymer compositions and different sizes in micron scale. The compression forcedisplacement behaviors were established and the stress-strain relationships were analyzed to examine the material response of polymer particles at different strain levels.

\section{MATERIALS AND METHODS}

\section{Apparatus}

The mechanical testing of single polymer particles was performed by using a nanomechanical testing system (TriboIndenter ${ }^{\circledR}$ Hysitron, MN), which is capable of operating in load or displacement controlled modes with load and displacement noise floor of $100 \mathrm{nN}$ and $1 \mathrm{~nm}$, respectively. During indentation, the force-displacement curves of tested materials are recorded. Nanoindentation-based flat punch method was developed to characterize an individual particle and a schematic figure is shown in Figure 1(a). A square diamond flat punch with $100-\mu \mathrm{m}$ sides was specially designed to compress polymer particles. The flat punch requires precise calibration, especially with respect to coplanarity. The flat punch was first cleaned to remove defects such as dust or external impurities. The coplanarity of the flat punch was calibrated by indents into a polished indium surface. A clear impression on the indium surface was required for the flat punch to be

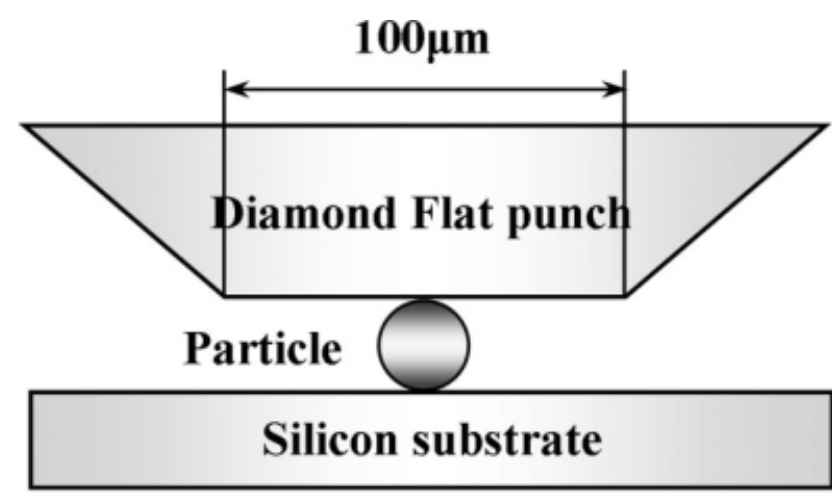

(a)

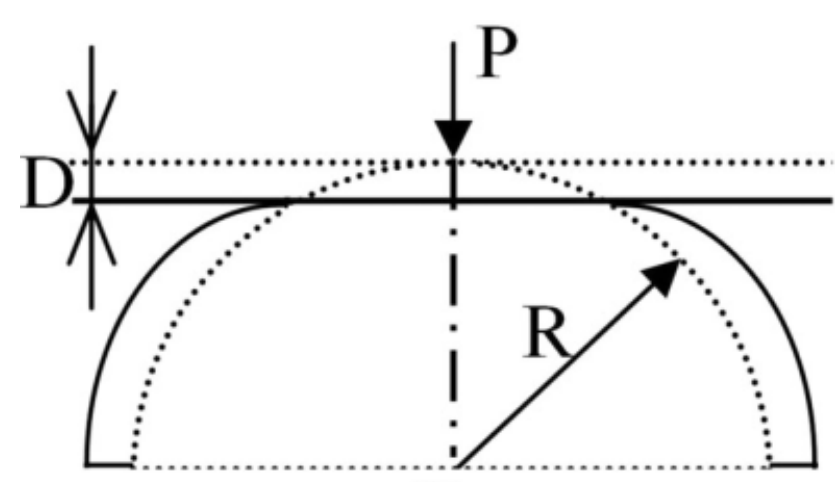

(b)

Figure 1 Schematic plots of the flat punch test (a) and model description (b).

acceptable. Also, the relative position between the integrated optical microscope and the flat punch is evaluated in the same way to calibrate the in situ test setting. By use of the optical microscope, single particles with more than $75-\mu \mathrm{m}$ distance to the closest neighbor was selected for the test. The single particle was then compressed between the diamond flat punch and the silicon substrate.

\section{Materials}

The commercially available acrylic copolymer (AC) particles (Concore ${ }^{\mathrm{TM}}$, Conpart AS, NO) and polystyrene (PS) particles (Dynospheres ${ }^{\circledR}$, Invitrogen Dynal AS, NO) were tested. Both particles were synthesized by an activated swelling method developed by Ugelstad, which produces highly mono-sized particles. ${ }^{25}$ The coefficient of variance $(\mathrm{CV})$ of the particle size distribution is less than $2 \%$, where $\mathrm{CV}$ is defined as the ratio of the standard deviation to the mean diameter. The AC particles are strongly crosslinked, whereas the PS particles are slightly crosslinked with divinylbenzene (DVB). The particles have an amorphous structure at room temperature. The glass transition temperatures have been estimated on the basis of the chemical compositions of the particles. The particles and their physical 
TABLE I

The Physical Properties of the Tested Particles

\begin{tabular}{lcccccc}
\hline & \multirow{2}{*}{$\begin{array}{c}\text { Diameter } \\
\text { Particle }\end{array}$} & $\begin{array}{c}\text { Estimated } \\
T_{g}(\mu \mathrm{C})\end{array}$ & Diacrylic & Acrylic & Styrene & DVB \\
\cline { 4 - 7 } AC1 & 3.0 & 42 & 60 & 40 & - & - \\
AC2 & 3.8 & 42 & 60 & 40 & - & - \\
AC3 & 4.8 & 42 & 60 & 40 & - & - \\
PS1 & 2.6 & 100 & - & - & 98 & 2 \\
PS2 & 5.1 & 100 & - & - & 98 & 2 \\
\hline
\end{tabular}

properties are given in Table I. AC1, AC2, and AC3 have identical chemical compositions but different sizes, and the same is true for particle PS1 and PS2.

During the sample preparation, a very small amount of the polymer particles were immersed in 95\% industrial ethanol and exposed to a high-frequency ultrasonic vibration to redisperse the particle clusters and to minimize the liquid surface tension effect. A droplet of the ethanol-particle suspension was placed onto a bare silicon chip $(10 \mathrm{~mm} \times 10$ $\mathrm{mm} \times 0.5 \mathrm{~mm}$ ). The specimen was then left to dry in a clean environment for a specific period of time to remove any ethanol left in the particles. It was easy to distinguish single particles from a cluster of two or more particles by using the attached optical microscope in the Triboindenter.

\section{Method}

Figure 1 shows the contact between a diamond flat punch and a single polymer particle. All tests were performed in air and at room temperature $\left(23^{\circ} \mathrm{C}\right)$. The room humidity was kept constant about 30\% through an air ventilation system. The standard load-controlled mode was selected for all particles in which the applied load is following a predefined load function. The load function with a peak load of $10 \mathrm{mN}$ and linear loading/unloading rate of $2 \mathrm{mN} / \mathrm{s}$ was used. A typical indentation was completed in $12 \mathrm{~s}$, which consists of a linear loading/unloading segment for $5 \mathrm{~s}$ and a $2 \mathrm{~s}$ load-holding segment at peak load. Before indentation started, the drift rate of the instrument was monitored for $40 \mathrm{~s}$ at a preload of $1 \mu \mathrm{N}$, and an average drift rate calculated by fitting a straight line to the drift displacement versus time during the last $20 \mathrm{~s}$ was used to correct the resulting data. Typically, small drift rates on the order of $0.1 \mathrm{~nm} / \mathrm{s}$ were observed. For each group of particles, at least four single particles have been tested to check the repeatability of the results.

\section{RESULTS AND DISCUSSION}

\section{Experimental results}

The loading and unloading behaviors of different indentations for each group of particles are found to be very consistent. Even the fracture of the smallest AC particles occurs at the almost same load level each time. The indentation curves for single AC particles with identical chemical compositions, $60 \%$ diacrylate and $40 \%$ acrylate, $\mathrm{AC} 1, \mathrm{AC} 2$, and $\mathrm{AC} 3$, are shown in Figure 2. The particle AC1 shows destructive failure at a displacement of $1.84 \pm 0.02 \mu \mathrm{m}$, which corresponds to $61.3 \% \pm 0.7 \%$ deformation level. At this point, the particle immediately breaks down with a close to $20 \%$ increase in deformation. No failure points are observed for $\mathrm{AC} 2$ and $\mathrm{AC} 3$ under the $10-\mathrm{mN}$ peak load where maximum deformation for $\mathrm{AC} 2$ is $56.7 \% \pm 0.3 \%$ and for $\mathrm{AC} 3$ is $51.5 \% \pm 0.9 \%$, respectively. After unloading the displacement recoveries for $\mathrm{AC} 2$ and $\mathrm{AC} 3$ are around $47.3 \%$ and $54.7 \%$, which is defined as the ratio of the recovered displacement to the maximum displacement and represents the particle recoverability after being deformed by compression.

Another widely used polymer material, polystyrene particles, PS1 and PS2, are also tested. Two PS particles are made of same chemistry: $98 \%$ polystyrene slightly crosslinked with $2 \%$ divinylbenzene. The compression force-displacement curves for two single PS particles are plotted in Figure 3. Unlike the AC particles, the smaller PS particles do not show the explicit fracture behavior. However, there is

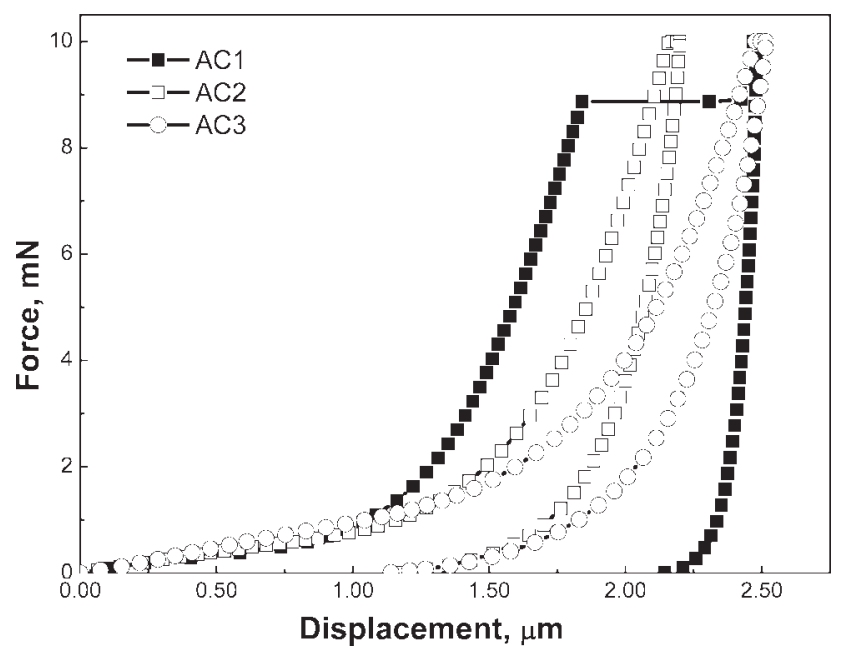

Figure 2 Representative flat punch test load-displacement curves of particle AC1, AC2, and AC3. 


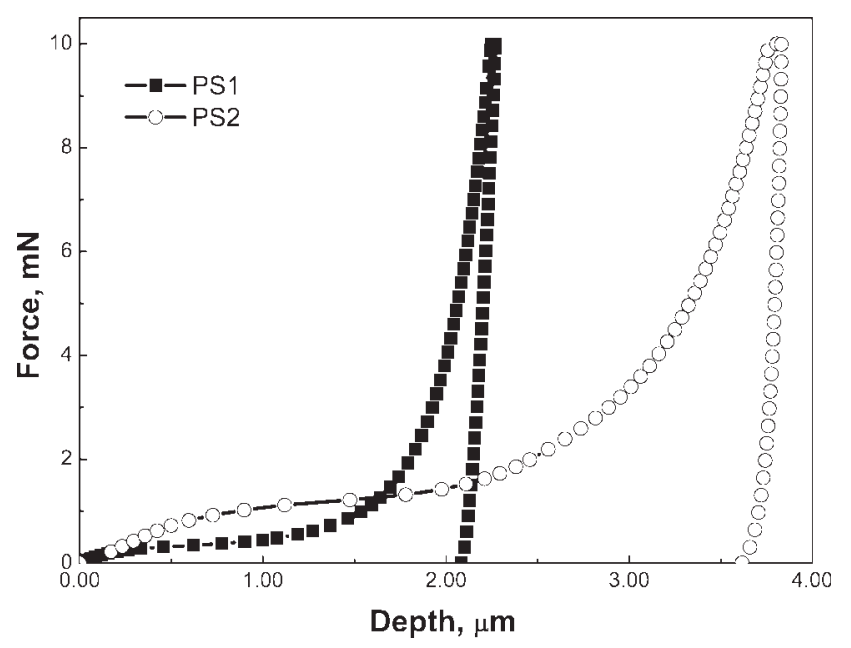

Figure 3 Representative flat punch test load-displacement curves of particle PS1 and PS2.

hardly any displacement recovery for either PS1 or PS2 after unloading. The maximum deformation at the $10-\mathrm{mN}$ peak load for PS1 and PS2 are $87 \% \pm$ $0.6 \%$ and $74.8 \% \pm 0.5 \%$, respectively.

\section{Stress-strain behaviors}

There are several analytical models describing the contact between a deformable sphere and a rigid flat. ${ }^{26}$ The well-known Hertz theory can be applied for small deformation contact of linear elastic materials and states that the contact force is proportional to the power 1.5 of the displacement. ${ }^{27,28}$ Hertz model is based on the following assumptions: the contact area between two elastic spheres is much smaller than the sphere size; the normal contact between two spheres is frictionless; and the stress distribution within the contact area is obtained by considering concentrated force applied to an elastic half-space and the effect of the sphere boundary on the contact deformation is neglected. For a somewhat larger deformation scale, Tatara theory could be used to predict the nonlinear elastic response of elastomeric spheres, which predicts the contact force-displacement relationship with the power of $3 . .^{29,30}$ Tatara theory can be considered as an extension of the Hertz model, which is the result of removing two of the main requirements for using Hertz theory, namely the small deformations and linear elasticity. However, Hertz and Tatara theories are still valid in small strain scale. Zhang's model, which is based on finite element analysis, introduces explicit solutions for the compression stress-strain relationship of linear elastic materials for large deformation. ${ }^{31}$ Zhang's model claims that the contact force is approximately proportional to the power 1.52 of the displacement. However, in this study a deformation above $50 \%$ has been reached and none of the existing theories are therefore applicable.
During indentation, both microstructure and geometry of particles influence the compression behavior. Therefore, normalization of the experimental results should be used to remove the effect of particle dimensions. The volume and Poisson's ratio of polymer particles might change continuously with the large deformation because of the polymer nature and the sphere geometry. ${ }^{32,33}$ It is impossible to obtain the true stress-strain behaviors of particles from the actual experiment. The nominal compression stress-strain relationships of the particles are therefore calculated based on the diameter and the cross-sectional area of the undeformed particle:

$$
\sigma_{N}=\frac{P}{\pi R^{2}} \text { and } \varepsilon_{N}=\frac{D}{R}
$$

where $P$ is the applied force, $D$ is the half-displacement, and $R$ is the initial particle radius, shown in Figure 1(b). The resulting stress-strain curves are plotted in Figure 4. The focus of this study is the

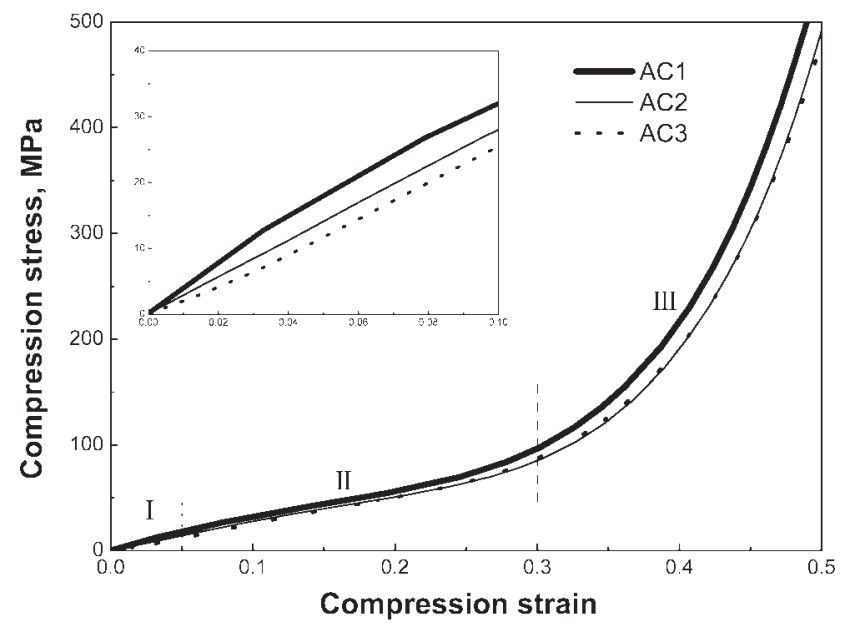

(a)

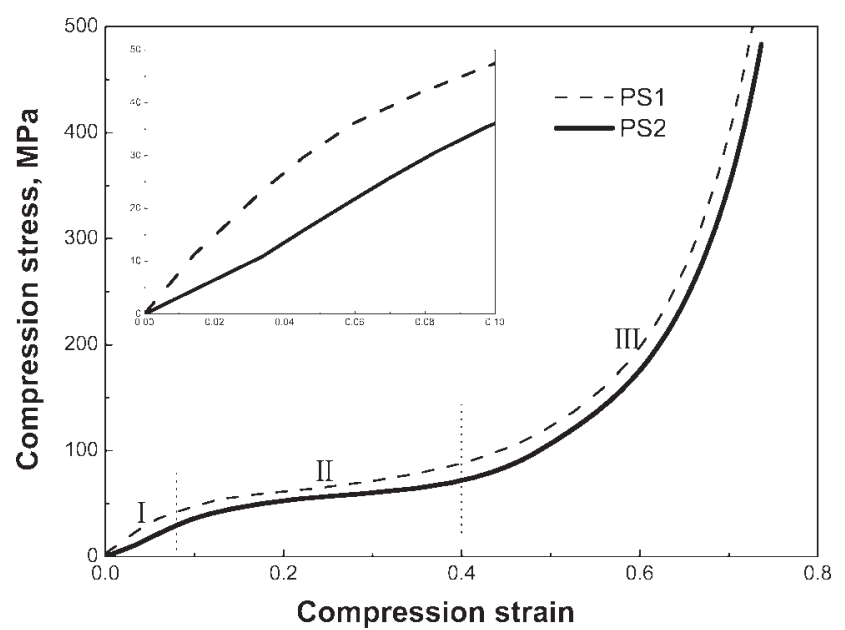

(b)

Figure 4 Compression stress-strain behaviors of polymer particle (a) AC1, AC2, and AC3 and (b) PS1 and PS2. 


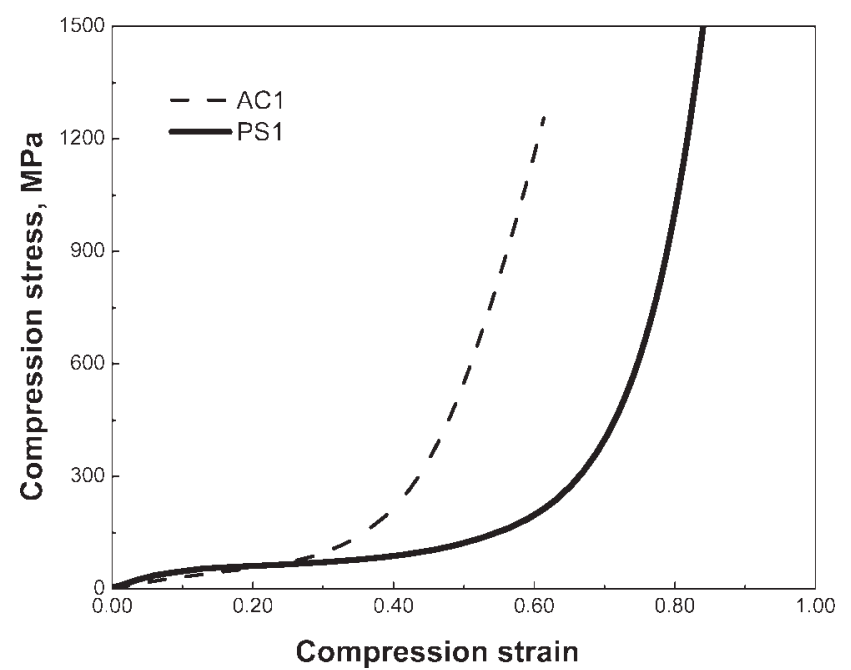

Figure 5 Comparison of AC and PS particles.

loading part and the unloading stress-strain curves are omitted.

A comparison of the AC particle and PS particle is shown in Figure 5, where the smaller particles AC1 and PS1 are plotted. The stress-strain curve of the AC particle appears to rise monotonically. The PS particle displays a longer plateau than the AC particle, which means that the PS particle has better deformation resistance at lower stress level. The AC particle behaves softer than the PS particle when the strain is less than $22 \%$. The AC particle therefore shows more brittle behavior than the PS particle.

According to Zhang et al.'s model, ${ }^{31}$ the compression stress-strain behavior for any given Poisson's ratio can be described as:

$$
\sigma_{C}=\left(\frac{\sigma_{C}}{K}\right)_{v=0.3} K_{v}
$$

where $\left(\frac{\sigma_{C}}{K}\right)_{v=0.3}$ is the finite element solution with Poisson's ratio of 0.3 and $K_{v}$ is the reference compression modulus. It has been shown that for the cases with larger Poisson's ratio $(v>0.3)$ involved, the reference modulus is a good representation of the actual compression modulus.

The finite element solution for the case with Poisson's ratio of 0.3 has been fitted by a cubic polynomial:

$$
\begin{array}{r}
\left(\frac{\sigma_{C}}{K}\right)_{v=0.3}=0.033 \varepsilon_{C}+0.99 \varepsilon_{C}^{2}-1.122 \varepsilon_{C}^{3}, \\
\text { for } 1 \% \leq \varepsilon_{C} \leq 10 \%,
\end{array}
$$

$$
\begin{array}{r}
\left(\frac{\sigma_{C}}{K}\right)_{v=0.3}=0.0667 \varepsilon_{C}+0.5105 \varepsilon_{C}^{2}+0.5724 \varepsilon_{C}^{3}, \\
\text { for } 10 \%<t \varepsilon_{C} \leq 20 \% .
\end{array}
$$

When the deformation rises over $20 \%$, there is no unique solution for particle compression and the finite element results for each group of particles have to be considered separately. The reference compression modulus $K_{v}$ with $v=0.3$ for the particles is shown in Figure 6 for a range up to $40 \%$ strain. The compression modulus of the PS particles is more evenly distributed than the AC particles. The compression modulus of the PS particles steadily decreases in the strain interval from 1 to $40 \%$. This can possibly be explained by local plastic flow as well as observed surface cracking in the material.

To investigate the stress-strain behaviors of amorphous polymers, the most reliable method is uniaxial compression or shear measurement, to avoid the craze formation that can occur in tensile tests. Depending on materials and deformation conditions such as strain rate and temperature, the amorphous polymer usually contains three types of nonuniform deformation processes, which are yield behavior, rubber-like behavior, and brittle fracture. ${ }^{34-36}$ Yield behavior exhibits an initially linear portion corresponding to purely elastic behavior. With increasing stress, a nonlinear elastic behavior occurs where the strain goes back to zero after unloading, but along a curve slightly lower than the linear elastic one. A yielding and strain softening portion is observed, representing the presence of a permanent deformation. At a large strain level, polymer begins to show strain hardening, which is evident by a dramatic upturn in the stress-strain curve. For rubber-like behavior, the plastic flow occurs at the same stress level as that required for the yielding so the strain softening does not exist. The polymer is a long plastic flow process due to the stretch of long chains. In the case of brittle fracture behavior, the strain hardening happens very close to yield point or even no clear yield point exists, resulting from the suppressed strain softening and plastic flow.

In the compression stress-strain curves for the three AC particles in Figure 4(a), it is important to

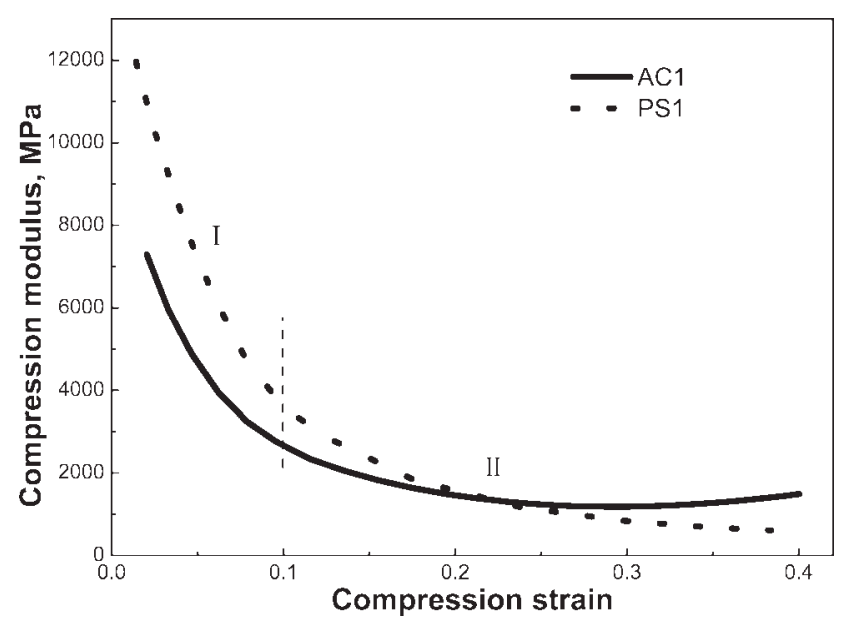

Figure 6 Estimated reference compression modulus. 
note that there is no clear yielding evidence and no presence of plastic flow. The AC particles behave more or less as a brittle fracture. The AC particles first possess a stiff response shown as zone $I$ in Figure 4(a). At zone II, the particles exhibit a nonlinear deformation in which particles reach yielding and have a continuous process of local configurational rearrangements of molecular segments. ${ }^{32,37} \mathrm{At}$ a higher strain level around 30\%, an abrupt increase of stress occurs, which represents the strain hardening in zone III. Finally, the smallest particle, AC1, collapses at around $61 \%$ strain, whereas the larger particles, AC2 and AC3, deform up to 56 and $51 \%$ strain. Unlike the AC particles, the PS particles agree with the rubber-like behavior pattern, seen in Figure 4(b). In the stress-strain curves the PS particles have an initially stiff response as zone I, the definite yielding point, long plateau implying the plastic flow behavior within zone II and the final strain hardening in zone III. The larger particle PS1 displays a plateau from 20 to $40 \%$ strain and the smaller particle PS2 exhibits even longer plateau of plastic flow from 15 to $38 \%$ strain. The PS2 deforms up to $80 \%$ without showing any critical fracture point. In Figure 5 the stress-strain behaviors of two types of particles are plotted and the difference between them is very clear. The PS particles experience a high degree of deformation with a long plateau, while the AC particles rise continuously.

In Figure 6 the compression modulus is considered as occurring in two zones according to the proceeding analysis. In zone I the compression modulus of particles decreases very fast within $10 \%$ compression strain. When the compression strain is above $10 \%$, as shown in zone II, the decrease of the compression modulus becomes slower and tends to be constant. At the small strain level, the strain rate is much higher than the large strain level. In this case the deformation of particles is dominated by viscosity, through resisting shear flow. As the strain increases, the strain rate decreases gradually and the effect of viscosity becomes weaker and weaker.

\section{Size effect}

The stress-strain relationship is one of the constitutive properties of materials. From the continuum mechanics point of view, for a specific material with different specimen dimensions but same chemistry, all stress-strain curves should collapse into one. However, in Figure 4(a,b), a particle size effect can be clearly observed for both AC and PS particles in the embedded diagrams. For the AC particles in Figure 4(a), the compressive stress-strain behaviors are particle size dependent; the smaller the diameter is, the harder the particles behave. The smallest par-

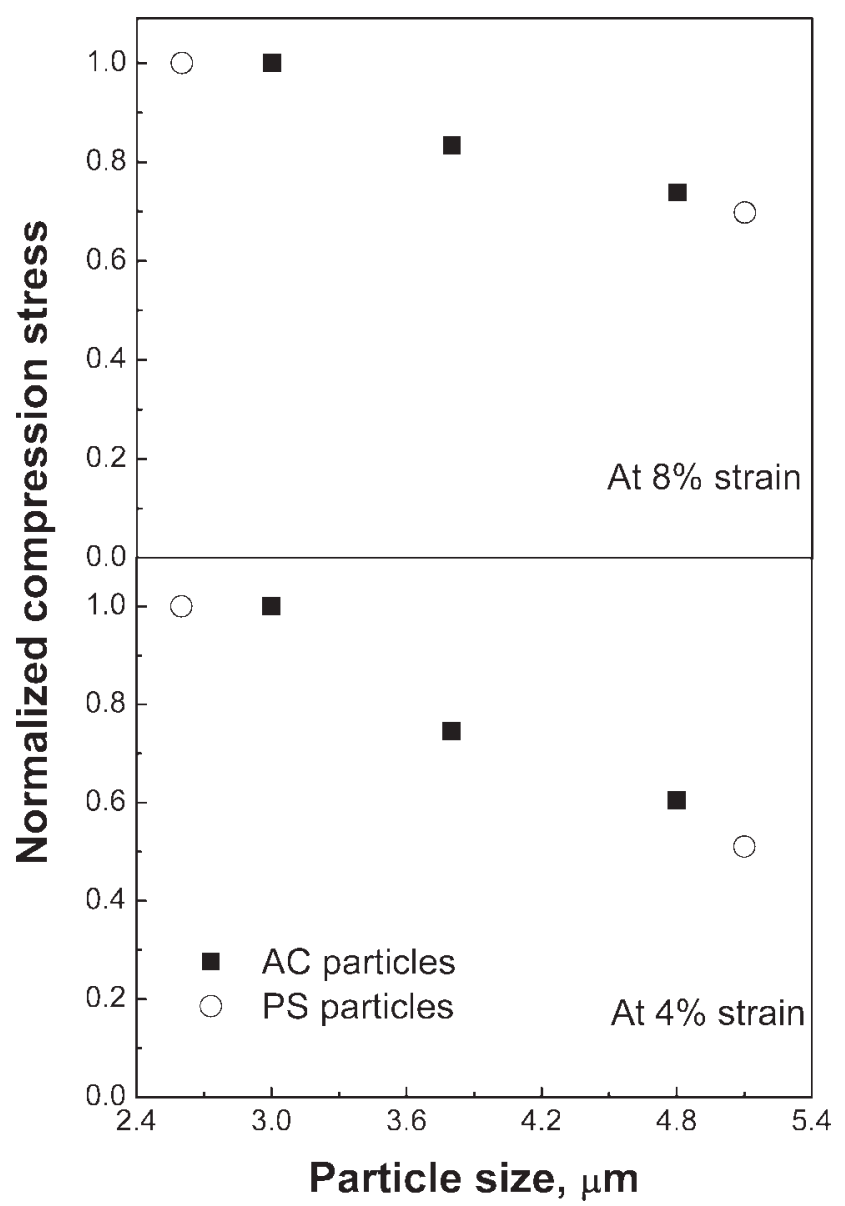

Figure 7 Particle size dependence of the normalized stress.

ticle $\mathrm{AC} 1$ is the hardest, while the biggest $\mathrm{AC} 3$ is the softest. In Figure 4(b), for the PS particles, the size dependence of the stress-strain behaviors becomes even more pronounced.

The nominal compression stress of the AC and PS particles at 4 and $8 \%$ deformation levels are plotted in Figure 7, in which the compression stress is normalized by the corresponding value of the smallest particle $\mathrm{AC} 1$ for $\mathrm{AC}$ particles and the smaller particle PS1 for PS particles. At 4\% strain level, the compression stress of the particle AC3 is almost $40 \%$ lower than that of the particle AC1; PS2 is even $50 \%$ lower than PS1. Increasing strain to $8 \%$, the particle size-dependence becomes weaker, which shows that the difference between $\mathrm{AC} 1$ and $\mathrm{AC} 3$ decreases to $26 \%$ and it is $30 \%$ between PS1 and PS2. The trend of particle size-dependent stress is consistent with the previous findings, where constant displacement rates and constant deformation levels were used. $^{38}$

In the present study the instrument operates with a constant loading rate and peak load, which means that the strain rate is significantly reduced as the displacement increases. This can possibly be 
interpreted by a viscous material model where the viscous effects are significant at low contact strain. The nonuniform strain rate can possibly cause the different viscous response, resulting in the dependence of the stress-strain behaviors on the particle size. During the synthesis of the polymer particles, the crosslinking monomer diffuses through the water phase and into the polymer seeds, causing it to expand. ${ }^{25}$ After polymerization, the distribution of the crosslinking monomer inside the polymer particles is determined by diffusion conditions such as monomer concentration in solvent, temperature, monomer hydrophilicity, and reaction time. A conceivable inhomogeneity of crosslink distribution can induce uneven microstructure, which might influence mechanical behaviors of the polymer particles. The particle size effect can also be induced by a prestrain due to the liquid surface tension and the adhesion between particles and the silicon chip or the diamond flat punch. The prestrain has a relatively significant effect on the smaller particles than the larger particles. Also the energy imposed onto the particles during indentation can cause temperature effects that can alter the mechanical properties of the polymer particles. There is a significant hysteresis in the loading-unloading curve, which suggests that mechanical energy is transformed into heat and therefore leads to a temperature rise of the particles. Within the time scale of the experiment, some of this thermal energy is dissipated to the silicon chip and the diamond flat punch. The thermal effect is varied with the different particle sizes. The strong temperature dependence of mechanical properties then leads to the variation of the stress-strain behaviors.

\section{CONCLUSION}

An experimental investigation of the mechanical behaviors of micron-sized polymer particles is performed by using the nanoindentation-based flat punch method. The following conclusions can be drawn from the presented results and interpretations.

The compression force-displacement behaviors of two polymer particles (acrylic and polystyrene) have been established by using nanoindentation-based flat punch technique. It has been demonstrated that it is possible to distinguish the mechanical properties of different polymer particles from indentation results. The evident fracture of polymer particles can be observed directly in the force-displacement curves.

Comparing the stress-strain relationships of AC and PS particles, the AC particles show brittle fracture behavior, while the polystyrene particles comply with yield behavior. The smaller particles of both types of polymers display more distinct viscosity than the larger particles.

A particle size effect on the stress-strain behaviors is observed on both polymer particles: the smaller the diameter, the harder the particles. The size-dependence of the compression stress is reduced when the strain increases. Further understanding of the particle size-dependent mechanisms is required.

The results can be used to design particle properties for ACA assemblies.

\section{References}

1. Ahmad, H. Colloid Polym Sci 2003, 281, 476.

2. Miled, K.; Sab, K.; Roy, R. L. Mech Mater 2007, 39, 222.

3. Cook, G.; Rudin, A.; Plumtree, A. J Appl Polym Sci 1993, 48, 75 .

4. Dagli, G.; Argon, A. S.; Cohen, R. E. Polymer 1995, 36, 2173.

5. Alfarraj, A.; Nauman, E. B. Polymer 2004, 45, 8435.

6. Kuboki, T.; Jar, P. Y. B.; Takahashi, K.; Shinmura, T. Macromolecules 2002, 35, 3584.

7. Lai, Z.; Liu, J. IEEE T Compon Pack T 1996, 19, 644.

8. Kristiansen, H.; Liu, J. IEEE T Compon Pack T 1998, 21, 208.

9. Liu, J.; Tolvgård, A.; Malmodin, J.; Lai, Z. IEEE T Compon Pack T 1999, 22, 186.

10. Kristiansen, H.; Gronlund, T.; Liu, J. In Proceedings of the 6th IEEE CPMT HDP'4; 2004; p 259.

11. Paik, K. W.; Kwon, W. S. In Proceedings of 10th International Symposium on Advanced Packaging Materials (IEEE), Processes, Properties and Interfaces; 2005; p 214.

12. Kim, D. O.; Jin, J. H.; Shon, W. I.; Oh, S. H. J Appl Polym Sci 2007, 105, 585.

13. Galloway, J.; Syed, A.; Kang, W.; Kim, J. Y.; Cannis, J.; Ka, Y. H. IEEE T Compon Pack T 2005, 28, 297.

14. Dou, G.; Whalley, D.; Liu, C. In Proceedings of IEEE 56th Electronic Components and Technology Conference; 2006; p 932.

15. Kwon, W. S.; Paik, K. W. IEEE T Compon Pack T 2006, 29, 528.

16. Kristiansen, H.; Zhang, Z. L.; Liu, J. In Proceedings of 10th International Symposium on Advanced Packaging Materials (IEEE), Processes, Properties and Interfaces; 2005; p 209.

17. Chin, M.; Iyer, K. A.; Hu, S. J. IEEE T Compon Pack T 2004, $27,317$.

18. Yim, M. J.; Kim, H. J.; Chung, C. K.; Paik, K. W. In Proceedings of IEEE 56th Electronic Components and Technology Conference; 2006; p 338.

19. Wang, X.; Wang, Y.; Chen, G.; Liu, J.; Lai, Z. IEEE T Compon Pack T 1998, 21, 248.

20. Fu, Y.; Wang, Y.; Wang, X.; Liu, J.; Lai, Z.; Chen, G.; Willander, M. IEEE T Adv Packaging 2000, 23, 15.

21. He, J. Y.; Zhang, Z. L.; Kristiansen, H. Int J Mater Res 2007, $98,389$.

22. Kim, D. O.; Jin, J. H. J Appl Polym Sci 2007, 105, 783.

23. Kim, D. O.; Jin, J. H. J Appl Polym Sci 2007, 104, 2350.

24. Dou, G.; Whalley, D. C.; Liu, C. Appl Phys Lett 2008, 92, 104108.

25. Ugelstad, J.; Berge, A.; Ellingsen, T.; Schmid, R.; Nilsen, T. N.; Mork, P. C. Prog Polym Sci 1992, 17, 87.

26. Liu, K. K. J Phys D Appl Phys 2006, 39, R189. 
27. Johnson, K. L. Contact Mechanics; University Press: Cambridge, 2003.

28. Liu, K. K.; Williams, D. R.; Briscoe, B. J. J Phys D Appl Phys 1998, 31, 294.

29. Tatara, Y. J Eng Mater Tech ASME 1989, 111, 163.

30. Tatara, Y. J Eng Mater Tech ASME 1991, 113, 285.

31. Zhang, Z. L.; Kristiansen, H.; Liu, J. Comput Mater Sci 2007, $39,305$.

32. Chow, T. S. Macromolecules 1992, 25, 440.

33. Li, Y.; Hu, Z.; Li, C. J Appl Polym Sci 1993, 50, 1107.
34. Chow, T. S. J Rheol 1992, 36, 1707.

35. Monnerie, L.; Halary, J. L.; Kausch, H. H. Adv Polym Sci 2005, 187, 215.

36. Strobl, G. The Physics of Polymers, 3rd ed.; Springer: Berlin, Heidelberg, 2007

37. Klapperich, C.; Komvopoulos, K.; Pruitt, L. J Tribol 2001, 123, 624.

38. He, J. Y.; Zhang, Z. L.; Midttun, M.; Fonnum, G.; Modahl, G. I.; Kristiansen, H.; Redford, K. Polymer 2008, 49, 3993. 